DOSSIER

\title{
Management et santé
}

sous la direction de

Mathieu Detchessahar 


\section{Le rédacteur invité, Mathieu Detchessahar, et la rédaction de la Revue française de gestion remercient les évaluateurs de ce dossier}

Emmanuel Abord de Chatillon, Université de Savoie

Cécile Clergeau, Université d'Angers

Mathieu Detchessahar, Université de Nantes

Michel Devigne, École des mines de Nantes

Johanna HABIB, Université de Nantes

Lionel Honoré, Sciences Politiques Rennes

Benoit JouRnÉ, Université du Maine

Cathy Krohmer, Université de Nantes

Laetitia Pinel, Université de Nantes

Gwenaelle Poilpot-Rocaboy, Université de Rennes I 
MATHIEU DETCHESSAHAR

Université de Nantes .

\section{Management et santé}

$\mathrm{N}$ ombre de pays post-industrialisés rencontrent, dès le début des années 1990, une épidémie énigmatique: l'expansion rapide des maux de dos et des tendinites qui conduit à une forte inflation des troubles-musculo-squelettiques (TMS) désormais inscrits au tableau des maladies professionnelles. Plus récemment, c'est l'augmentation des plaintes subjectives liées au travail qui occupe le devant de la scène et alimente le débat public: stress, burn-out, suicides, etc. Toutes les catégories de salariés semblent touchées dans de nombreux et différents secteurs d'activité, et les cadres n'échappent pas, loin s'en faut, au phénomène. On comprend dans ces conditions que la santé au travail soit devenue en quelques années un thème central de l'espace public français, un terrain important d'action gouvernementale et un sujet de négociation sociale dans les entreprises.
Face à ces nouvelles pathologies du travail, l'analyse des accords signés dans les entreprises de plus de 1000 salariés (Direction générale du travail, 2011) montre que deux voies d'actions semblent pour le moment privilégiées.

D'un côté, et conformément aux préconisations du rapport Légeron Nasse (2008), une partie de l'attention et des moyens se concentre sur la mesure du phénomène. On voit ainsi se multiplier les observatoires des risques psychosociaux en entreprise et les enquêtes diligentées par les pouvoirs publics. En ce qui concerne l'entreprise, les démarches de diagnostic sont présentes dans tous les accords et s'accompagnent le plus souvent de la création de groupes ad hoc qui ont vocation à piloter les études, dont une part importante est souvent constituée d'une enquête par questionnaire. D'un autre côté, l'action des directions d'entreprise se déploie pour une bonne part 
au niveau individuel. Il s'agit alors de mieux accompagner les individus en difficulté par des dispositifs leur permettant de recomposer leurs ressources physiques et subjectives (cellules d'écoute, groupes de parole, coaching, formation " gestes et postures ", massages, etc.) avant de les renvoyer vers une situation de travail qui demeure, elle, inchangée. L'analyse des accords signés dans les entreprises de plus de 1000 salariés montre à nouveau que des mesures comme la formation des managers, notamment à la détection des salariés en difficulté, la mise en place de procédures d'alerte ou de cellules d'écoute psychologique tiennent une place importante dans les voies d'action envisagées.

On constate à quel point, quand il s'agit de passer à l'action concrète, les directions comme les syndicats ont bien du mal à se saisir des questions d'organisation du travail et de management sur lesquelles pourtant d'autres textes, à vocation il est vrai plus programmatique (l'ANI de 2008 ou encore le rapport Lachmann), insistent tant. Ainsi le rapport de la DGT souligne que « quand on touche au partage du sens du travail, à la place du collectif, à l'organisation du travail et à la ligne managériale (...), très peu d'accords explorent cette piste sous toutes ces dimensions... ». Or, c'est précisément sur ces thèmes d'essence éminemment managériale que ce dossier de la Revue française de gestion entend se concentrer. Qu'ont à dire les sciences de gestion sur les liens unissant management et santé au travail et sur les voies d'action pertinentes dans ce domaine? Différentes réponses seront apportées ici. Elles sont proposées par des chercheurs qui partagent une commune pratique de la recherche-intervention et ont donc été confrontés sur leurs terrains de recherche à la double question de la compréhension du milieu dans lequel ils interviennent et de l'action dans et sur ce milieu.

Le premier texte est tout entier tourné vers la question de la conception d'organisations qui soutiendraient des dynamiques psychosociales positives. Cette démarche de (re)conception de l'organisation s'appuie sur un modèle d'analyse qui met les phénomènes identitaires au cœur de la vie des organisations. Ce modèle, Jean-Claude Sardas, Cédric Dalmasso et Philippe Lefebvre l'appellent le modèle DIGA: dynamique identitaire globale de l'acteur. Il s'agit d'une tentative originale, ambitieuse et convaincante de penser en même temps les différentes dimensions des dynamiques identitaires (dimensions cognitive, physiologique, stratégique et subjective), de mettre à jour leurs liens avec la construction de la santé psychique au travail et de bâtir, sur ces fondations, une démarche d'intervention. L'ensemble du processus est illustré par un cas concret de recherche-intervention dans une grande entreprise industrielle aux prises avec les problèmes d'internationalisation de ses activités d'ingénierie.

L'article de Mathieu Detchessahar propose une synthèse des principaux résultats obtenus au terme d'un programme de recherche financé par l'Agence nationale de la recherche, conduit par un collectif de chercheurs de différentes disciplines et ayant réuni jusqu'à aujourd'hui une vingtaine d'organisations autour de la question des déterminants organisationnels et managériaux de la santé au travail. Cette recherche montre que, à rebours des représentations de sens commun, les salariés des organisations étudiées, loin de souffrir des excès ou de l'omniprésence du management, pâtissent à l'inverse de l'absence de manage- 
ment. La recherche dresse le constat d'un management de proximité empêché, happé par les directions et attiré vers d'autres scènes que celles de la régulation du travail afin, notamment, de répondre aux exigences d'un gouvernement de l'entreprise de plus en plus complexe. Dans ces conditions, agir sur le risque de santé suppose de " désempêcher " le management de proximité de manière à le remettre au cœur des espaces de discussion du travail dont il faut penser l'ingénierie. L'article se conclut sur quelques réflexions ayant trait à la conception de ces espaces de discussion.

Le texte de Pierre-Yves Gomez et Romain Chevallet s'intéresse aux impacts des technologies de l'information sur la santé au travail, thème qui a pour tout praticien de l'entreprise un certain caractère d'évidence et qui, pourtant, a été finalement peu traité par la littérature spécialisée, notamment en systèmes d'information. L'article part d'un paradoxe: comment expliquer que la tertiarisation de nos économies, qui repose pour une bonne part sur une vague importante d'informatisation puis sur un usage extensif des technologies de l'information et de la communication technologies a priori plus « douces » que celles de l'ère industrielle, s'accompagne d'une montée des plaintes concernant les conditions de travail? Cette question est instruite à partir d'une recherche menée sur la plate-forme logistique d'un grand distributeur à l'occasion de l'introduction d'un système de guidage vocal pour soutenir le travail de manutention. Ce terrain est passé par les chercheurs au crible de différentes hypothèses concernant les effets de pénibilité directe de la technologie, les effets d'intensification du travail et les impacts en termes de rationalisation et de perte d'autonomie des acteurs.
La recherche conduit à insister tout particulièrement sur les deux derniers phénomènes ainsi que sur la façon dont les préparateurs de commande y font face et livre « un combat contre la perte de sens $»$. Tout en refusant le déterminisme technologique, les auteurs insistent sur l'absence de neutralité de la technologie et la façon dont elle pèse sur les usages.

Thierry Bertrand et Arnaud Stimec s'interrogent, dans le quatrième article, sur les effets sur la santé des pratiques de management issues du modèle du lean management, modèle qui semble s'instituer, depuis plusieurs années dans l'industrie, et plus récemment dans les grandes entreprises de service, comme le nouveau modèle standard d'organisation. Leur réflexion est nourrie par un important travail de terrain conduit dans trois sites industriels différents ayant articulé leurs récentes réorganisation autour des pratiques du lean. Leur recherche fait apparaître un bilan de santé contrasté du modèle du lean management. La qualité de l'accompagnement du nouveau modèle est bien entendu centrale, particulièrement la capacité du management de l'entreprise à faire vivre des dynamiques communicationnelles autour du travail. La qualité des espaces de discussion formels et informels, au sein desquels l'activité concrète peut être mise en débat, apparaît comme une pratique modératrice des effets du lean sur la santé.

Pour conclure ce dossier, Guy Minguet nous invite à grimper dans l'échelle hiérarchique pour interroger, en amont des activités opérationnelles, l'impact du travail des directions sur la santé des salariés. L'article étudie en effet, sur deux sites industriels, le lien existant entre la construction de la santé des salariés et différentes modalités 
" d'action dirigeante ». La recherche montre que la santé, dans ses diverses expressions, peut-être soutenue ou non par l'action dirigeante, selon que celle-ci construit ou non des repères de proximité qui soutiennent l'efficacité opérationnelle comme les dynamiques identitaires et nourrit ou non des espaces de régulation qui serviront de creuset au projet collectif. Dans ce travail, c'est la légitimité institutionnelle et professionnelle des dirigeants qui est en jeu, non pas dans leur capacité à « mobiliser les salariés ", mais dans leur habileté à construire un espace accueillant à leur volonté de donner de leur professionnalisme. C'est, selon Guy Minguet, par ce pacte managérial révisé que « la santé peut revenir au travail ».

\section{BibliograPhIE}

Accord National Interprofessionnel sur le stress au travail, 2 juillet 2008.

Direction générale du travail, Synthèse de l'analyse des accords signés dans les entreprises de plus de 1000 salariés, ministère du Travail, de l'Emploi et de la Santé, avril 2011.

Lachman H. et al., Bien-être et efficacité au travail. Dix propositions pour améliorer la santé psychologique au travail, Rapport au Premier ministre, 2010.

Légeron P., Nasse P., Rapport sur la détermination, la mesure et le suivi des risques psychosociaux au travail, ministère du Travail, des Relations sociales et de la Solidarité, 42, 2008. 